\title{
Rank and the Drazin inverse in Banach algebras
}

\author{
by \\ R. M. Brits (Johannesburg), L. Lindeboom (Pretoria) \\ and H. Raubenheimer (Johannesburg)
}

\begin{abstract}
Let $A$ be an arbitrary, unital and semisimple Banach algebra with nonzero socle. We investigate the relationship between the spectral rank (defined by B. Aupetit and H. Mouton) and the Drazin index for elements belonging to the socle of $A$. In particular, we show that the results for the finite-dimensional case can be extended to the (infinitedimensional) socle of $A$.
\end{abstract}

1. Introduction. Throughout this paper we shall assume that $A$ is a semisimple, complex and unital Banach algebra with the unit denoted by 1 and the group of invertible elements by $A^{-1}$. We denote by $\exp (A)$ the set of all exponentials of $A$, that is, $\exp (A)=\left\{e^{x}: x \in A\right\}$. For $a \in A$ we denote the spectrum of $a$ by $\sigma(a, A)$, with the convention that we write $\sigma(a)$ if the algebra under discussion is clear from the context. The nonzero spectrum of $a \in A$ is denoted $\sigma^{\prime}(a)=\sigma(a) \backslash\{0\}$. An element $a \in A$ belongs to the socle of $A$, denoted $\operatorname{Soc}(A)$, if there exist finitely many minimal left ideals or, equivalently, minimal right ideals such that $a$ belongs to their sum. The set $\operatorname{Soc}(A)$ is a two-sided ideal of $A$. We assume $\operatorname{Soc}(A) \neq\{0\}$.

Following [3] we define the rank of an element $a \in A$ by

$$
\operatorname{rank}(a)=\sup _{x \in A} \# \sigma^{\prime}(x a) \leq \infty
$$

where \# denotes the number of elements in a set. By the semisimplicity of $A$ we know that $\operatorname{rank}(a)=0$ if and only if $a=0$. Notice further that for $a \in A$ we have $\operatorname{rank}(a) \leq \operatorname{rank}(1)$ with equality whenever $a \in A^{-1}$. It can be shown ([3, Corollary 2.9]) that $\operatorname{Soc}(A)=\{a \in A: \operatorname{rank}(a)<\infty\}$, the set of finite rank elements of $A$. Also, if $a \in \operatorname{Soc}(A)$ then ([3, Theorem 2.2]) the set

$$
E(a)=\left\{x \in A: \# \sigma^{\prime}(x a)=\operatorname{rank}(a)\right\}
$$

is a dense open subset of $A$. Amongst the elements of $\operatorname{Soc}(A)$ Aupetit and Mouton distinguish the maximal finite rank elements as those $a \in \operatorname{Soc}(A)$

2000 Mathematics Subject Classification: 46H05, 46H15, 47 A99.

Key words and phrases: rank, Drazin inverse, semisimple Banach algebra. 
with $\operatorname{rank}(a)=\# \sigma^{\prime}(a)$. Since the sets $E(a)$ are dense in $A$ the set of maximal finite rank elements is dense in $\operatorname{Soc}(A)$.

If $A$ is finite-dimensional then $A=\operatorname{Soc}(A)$, and conversely, if $A=\operatorname{Soc}(A)$ then $A$ is finite-dimensional. In general however, $\operatorname{Soc}(A)$ is not necessarily closed in $A$ and hence not necessarily finite-dimensional. A particularly good reason for studying this notion of (spectral) rank is that it coincides with the standard rank in the case $A=B(X)$, the Banach algebra of bounded linear operators on a Banach space $X$ ([3, p. 118]); for some pathologies occurring on a previous rank definition used by several other authors see [3, p. 124]. The results obtained in this paper support the evidence pointing to the spectral rank as a suitable notion of "rank" in the case of general semisimple Banach algebras. In particular, our main result, Theorem 2.7, extends the relationship between rank and Drazin index (defined hereafter), which is known to hold for complex matrices $\left(M_{n}(\mathbb{C})\right)$, to the socle of a semisimple Banach algebra.

An element $a \in A$ is said to be Drazin invertible in $A$ if there exist $b \in A$ and $k \in \mathbb{Z}^{+}$such that

(i) $a^{k} b a=a^{k}$,

(ii) $b a b=b$,

(iii) $b a=a b$.

The unique element ([11, Lemma 1]) $b$ satisfying (i)-(iii) is called the Drazin inverse of $a$. As is customary we denote the Drazin inverse of $a$ by $a^{D}$ and we call the least $k \in \mathbb{Z}^{+}$such that (i) holds the Drazin index of $a$. If for $a \neq 0$ we define $a^{0}=1$ then it is clear that $A^{-1}$ is precisely the subset of Drazin invertible elements with Drazin index zero. The subset of Drazin invertible elements with Drazin index equal to zero or one is called the group invertibles, where the term "group" refers to the fact that if $a$ is group invertible with group inverse $a^{D}$, then $\left\{a, a^{D}\right\}$ generates an abelian group with identity $a a^{D}$. Notice that if $a$ is Drazin invertible with Drazin inverse $a^{D}$ then $a^{D}$ is always group invertible (with group inverse $a^{2} a^{D}$ ), even though $a$ might not be group invertible. We denote the set of Drazin invertible elements by $\mathcal{D}(A)$ and the set of group invertibles by $\mathcal{G}(A)$. Although we restrict ourselves in this paper to the sets $\mathcal{D}(A)$ and $\mathcal{G}(A)$ we mention that the Drazin inverse belongs to the broad and intensively studied subject of generalized inverses, which is not confined only to the Banach algebra setting. For more on this topic (and some applications) see [6], [9].

In [5] it was shown that $\operatorname{Soc}(A) \subseteq \mathcal{D}(A)$ and that $\operatorname{Soc}(A)$ is the largest ideal contained in $\mathcal{D}(A)$. In fact, it was shown there that if $J$ is a left or right multiplicative ideal (i.e. $J$ only needs to absorb products in $A$ from the left or the right) such that $J \subseteq \mathcal{D}(A)$ then $J \subseteq \operatorname{Soc}(A)$. Since every maximal finite rank element can be written as a linear combination of orthogonal 
rank 1 idempotents ([3, Theorem 2.8]) it follows that the maximal finite rank elements are all group invertible in $\operatorname{Soc}(A)$. Hence $\operatorname{Soc}(A) \cap \mathcal{G}(A)$ is dense in $\operatorname{Soc}(A)$. In general (even for finite-dimensional cases) it is not true that every element of $\operatorname{Soc}(A)$ is group invertible; it was shown in [5] that $\operatorname{Soc}(A) \subseteq \mathcal{G}(A)$ if and only if every element of $\operatorname{Soc}(A)$ belongs to the center of $A$. Using generalized inverses, we show here (Corollary 2.2 (ii) $\Leftrightarrow(i i i)$ ) that the property "every element of $\operatorname{Soc}(A)$ is central in $A$ " is equivalent to an apparently much weaker condition on $\operatorname{Soc}(A)$.

In order to prove Theorem 2.7 we need a structural characterization of Aupetit and Mouton's spectral definition of rank for elements belonging to $\operatorname{Soc}(A)$. We start with some terminology and notation.

An element of an algebra $A$ is indecomposable if it cannot be written as a sum $a=b+c$ where $b, c \in A$ are nonzero elements satisfying $b A c=0$.

We denote by $M_{r, n}$, with $r \leq n \leq 2 r$, the algebra of all $n \times n$ complex matrices $\left[a_{i, j}\right]$ satisfying $a_{i, j}=0$ whenever $i>r$ or $j \leq n-r$.

We can now state the required result which we shall refer to as the rank structure decomposition (see [4, p. 289]) of an element belonging to $\operatorname{Soc}(A)$.

Theorem 1.1 (M. Brešar, P. Šemrl). Let $A$ be a semisimple unital complex Banach algebra and let $n \in \mathbb{N}$. Then $a \in A$ has $\operatorname{rank}(a)=n$ if and only if there exist $a_{1}, \ldots, a_{k} \in A$ such that

(i) $a=a_{1}+\cdots+a_{k}$,

(ii) each $a_{i}$ is indecomposable,

(iii) $a_{i} A a_{j}=0$ whenever $i \neq j$,

(iv) $a_{i} A a_{i} \simeq M_{r_{i}, n_{i}}$ for some $r_{i}, n_{i} \in \mathbb{N}, r_{i} \leq n_{i} \leq 2 r_{i}$,

(v) $n=r_{1}+\cdots+r_{k}$.

Moreover, $a_{1}, \ldots, a_{k}$ are unique nonzero elements in $\operatorname{Soc}(A)$ satisfying (i)-(iii).

As a first application of Theorem 1.1 we give an alternative proof of [3, Theorem 2.12] which is perhaps a bit easier and in fact proves a little more.

Theorem 1.2 (B. Aupetit, H. du T. Mouton). For $a \in A$,

$$
\operatorname{rank}(a) \leq \operatorname{dim}(a A a) \leq(\operatorname{rank}(a))^{2} .
$$

Moreover, if $a \in \operatorname{Soc}(A)$ then $\operatorname{rank}(a)=\operatorname{dim}(a A a)$ if and only if $a A a \simeq$ $M_{1, n_{1}} \oplus \cdots \oplus M_{1, n_{k}}$ where $n_{i} \in\{1,2\}$ and $k=\operatorname{rank}(a)$.

Proof. As in the first part of the proof of [3, Theorem 2.12] it suffices to consider $a \in \operatorname{Soc}(A)$. So let $a \in \operatorname{Soc}(A)$ with rank structure decomposition $a=a_{1}+\cdots+a_{k}$ and $\operatorname{rank}(a)=r_{1}+\cdots+r_{k}$ as in 1.1. For each $i$ we first prove

$$
a_{i} A a_{i} \cap\left(a_{1} A a_{1}+\cdots+a_{i-1} A a_{i-1}+a_{i+1} A a_{i+1}+\cdots+a_{k} A a_{k}\right)=\{0\} .
$$


If for some set $\left\{x_{1}, \ldots, x_{k}\right\} \in A$ we have

$$
a_{i} x_{i} a_{i}=\sum_{\substack{j=1 \\ j \neq i}}^{k} a_{j} x_{j} a_{j}
$$

then let $z \in A$ be such that $\operatorname{rank}\left(a_{i} x_{i} a_{i}\right)=\# \sigma^{\prime}\left(a_{i} x_{i} a_{i} z\right)$. Using $a_{i} A a_{j}=\{0\}$ we obtain

$$
\# \sigma^{\prime}\left(\left(a_{i} x_{i} a_{i} z\right)^{2}\right)=\# \sigma^{\prime}\left(a_{i} x_{i} a_{i} z\left(\sum_{\substack{j=1 \\ j \neq i}}^{k} a_{j} x_{j} a_{j} z\right)\right)=0
$$

and by the spectral mapping theorem it follows that $\# \sigma^{\prime}\left(a_{i} x_{i} a_{i} z\right)=0$. Thus $\operatorname{rank}\left(a_{i} x_{i} a_{i}\right)=0$ and hence $a_{i} x_{i} a_{i}=0$, which proves (1.2.1).

It follows further that

$$
a A a=\left(a_{1}+\cdots+a_{k}\right) A\left(a_{1}+\cdots+a_{k}\right) \subseteq a_{1} A a_{1}+\cdots+a_{k} A a_{k}
$$

where $\operatorname{dim}\left(a_{i} A a_{i}\right)=r_{i}^{2}$ for each $i$. Let $V_{i}=\left\{e_{1}^{(i)}, \ldots, e_{r_{i}^{2}}^{(i)}\right\}$ be a basis for $a_{i} A a_{i}$. Since $a_{i} A a_{i}$ is finite-dimensional one may easily prove that

$$
B_{i}=\left\{x \in A: a_{i} x a_{i}=\alpha_{1} e_{1}^{(i)}+\cdots+\alpha_{r_{i}^{2}} e_{r_{i}^{2}}^{(i)} \text { with } \alpha_{j} \neq 0 \text { for each } j\right\}
$$

is a dense and open subset of $A$. It follows from Baire's theorem that $\operatorname{dim}(a A a)=\operatorname{dim}\left(a_{1} A a_{1}+\cdots+a_{k} A a_{k}\right)$ and hence that

$$
a A a=a_{1} A a_{1}+\cdots+a_{k} A a_{k} .
$$

We thus have

$$
\begin{aligned}
\operatorname{rank}(a) & =r_{1}+\cdots+r_{k} \leq r_{1}^{2}+\cdots+r_{k}^{2} \\
& =\operatorname{dim}\left(a_{1} A a_{1}\right)+\cdots+\operatorname{dim}\left(a_{k} A a_{k}\right) \\
& =\operatorname{dim}\left(a_{1} A a_{1}+\cdots+a_{k} A a_{k}\right)=\operatorname{dim}(a A a) \\
& \leq\left(r_{1}+\cdots+r_{k}\right)^{2}=(\operatorname{rank}(a))^{2} .
\end{aligned}
$$

If $\operatorname{rank}(a)=\operatorname{dim}(a A a)$ then, following the above arguments, we deduce that $r_{i}=1$ for each $i$. Thus $a_{i} A a_{i}=\mathbb{C} a_{i} \simeq M_{1, r}$ where $r=2$ if $a_{i}$ is nilpotent and $r=1$ if $a_{i}$ is not nilpotent. The result follows by observing that (1.2.2) is actually a direct sum.

On the other hand, if $a A a \simeq M_{1, n_{1}} \oplus \cdots \oplus M_{1, n_{k}}$ where $n_{i} \in\{1,2\}$ and $k=\operatorname{rank}(a)$ then it follows directly that $\operatorname{dim}(a A a)=k=\operatorname{rank}(a)$.

The following lemma will be instrumental in some of our results and is a special case of [7, Theorem 6.4]. Note that, since every element of $\operatorname{Soc}(A)$ is algebraic, we can replace quasinilpotent in [7, Theorem 6.4] by nilpotent.

Lemma 1.3 (core-nilpotent decomposition). Let $a \in \operatorname{Soc}(A)$. Then a has a unique decomposition of the form $a=c_{a}+r_{a}$ where $c_{a}$ is a group invertible element in $\operatorname{Soc}(A), r_{a}$ is a nilpotent element in $\operatorname{Soc}(A)$ and $c_{a} r_{a}=r_{a} c_{a}=0$. 
REMARK. One may actually observe that, by the uniqueness of the corenilpotent decomposition, $c_{a}=a a^{D} a$ and $r_{a}=a\left(1-a a^{D}\right)$. The element $c_{a}$ is called the core of $a$ and $r_{a}$ the nilpotent part of $a$. The idempotent $1-a a^{D}$ is called the spectral projection of a corresponding to 0 ; the terminology being appropriate in view of the holomorphic functional calculus (see 1.6).

It is also appropriate, at this stage, to recall two facts that will be used throughout the remainder of this paper without specific reference:

(1.4) If $A$ is a semisimple Banach algebra with identity and $p \in A$ is an idempotent, then $p A p$ is a semisimple Banach algebra with identity $p$. Moreover, $\sigma^{\prime}(p x p, A)=\sigma^{\prime}(p x p, p A p)$ for every $x \in A$.

If $A$ is a Banach algebra and $a_{1}, \ldots, a_{n} \in A$ with $a_{i} a_{j}=a_{j} a_{i}=0$ for $i \neq j$, then $\sigma^{\prime}\left(a_{1}+\cdots+a_{n}\right)=\bigcup_{i=1}^{n} \sigma^{\prime}\left(a_{i}\right)$.

The first part of (1.4) and the containment $\sigma^{\prime}(p x p, p A p) \subseteq \sigma^{\prime}(p x p, A)$ are well known ([1, Lemma 2.5]). For the reverse containment notice that if $\lambda \neq 0$ and $p b p$ is the inverse to $\lambda p-p a p$ in $p A p$ then $p b p+\frac{1}{\lambda}(1-p)$ is the inverse to $\lambda$-pap in $A$. For (1.5), use induction on the argument in the proof of [3, Lemma 2.15]. Concerning (1.4), recall that if $p A p$ is finite-dimensional then the structure of $p A p$ is given by the Wedderburn-Artin theorem.

A particularly useful representation of the Drazin inverse is given by the holomorphic functional calculus: if $a \in \operatorname{Soc}(A)$ then 0 is an isolated point of $\sigma(a)$ or possibly, in the finite-dimensional case, $0 \notin \sigma(a)$. If the latter instance occurs then the Drazin inverse is just the usual inverse $a^{-1}$ and the representation follows easily. If 0 is isolated in $\sigma(a)$ then let $U_{0}$ be an open ball with center 0 and $U_{1}$ be an open set containing $\sigma(a) \backslash\{0\}$ such that $U_{0}$ and $U_{1}$ are separated in $\mathbb{C}$. Let $\Gamma_{0}$ be a circle in $U_{0}$ surrounding 0 and let $\Gamma_{1}$ be a smooth contour in $U_{1}$ surrounding $\sigma(a) \backslash\{0\}$. By the holomorphic functional calculus, the Drazin inverse of $a$ is given by

$$
a^{D}=\frac{1}{2 \pi i} \int_{\Gamma_{0} \cup \Gamma_{1}} g(\lambda)(\lambda-a)^{-1} d \lambda
$$

where

$$
g(\lambda)= \begin{cases}1 / \lambda, & \lambda \in U_{1} \\ 0, & \lambda \in U_{0}\end{cases}
$$

2. Rank and the Drazin inverse. For $a \in A$ we define the commutant of $a$ as the set $\operatorname{comm}(a)=\{x \in A: x a=a x\}$. If $B$ is a subset of $A$ and $a \in \operatorname{Soc}(A)$ then $a$ is said to assume its rank on $B$ if there is $b \in B$ such that $\operatorname{rank}(a)=\# \sigma^{\prime}(b a)$. Recall further that $x \in A$ is said to be central in $A$ if $x$ commutes with every element of $A$. As our first basic result, relating rank to group invertibility, we have 
Proposition 2.1. Let $a \in \operatorname{Soc}(A)$. If a assumes its rank on $\operatorname{comm}(a)$ then $a \in \mathcal{G}(A)$.

Proof. If $\operatorname{rank}(a)=0$ then $a=0$ and obviously the result holds. So let $a \in \operatorname{Soc}(A)$ with $\operatorname{rank}(a) \neq 0$ and let $u \in \operatorname{comm}(a)$ be such that $\operatorname{rank}(a)=$ $\# \sigma^{\prime}(u a)$. Now, since $a$ and $u$ commute, we cannot have $\sigma(a)=\{0\}$, and if $0 \notin \sigma(a)$ (which may happen in the finite-dimensional case) then the result follows trivially. Hence, using $2 \leq \# \sigma(a)<\infty$, it follows that

$$
l=\inf \{|\lambda-\alpha|: \lambda, \alpha \in \sigma(a), \lambda \neq \alpha\}>0 .
$$

Denote by $B$ the Banach algebra generated by $\{1, a, u\}$. Choose $\alpha \in \mathbb{C}$ with $|\alpha|>\max \left\{\|u\|, l^{-1} \operatorname{diam} \sigma(u a)\right\}$. For every complex homomorphism $\Phi$ on $B$ we have $\Phi(u a) \neq 0 \Rightarrow \Phi((\alpha-u) a) \neq 0$, thus establishing a correspondence $\sigma^{\prime}(u a) \mapsto \sigma^{\prime}((\alpha-u) a)$. Also, since $|\alpha|>l^{-1} \operatorname{diam} \sigma(u a)$, the correspondence is one-one. So there is $v \in \operatorname{comm}(a) \cap A^{-1}$ such that $\operatorname{rank}(a)=\# \sigma^{\prime}(a v)$. Moreover, it is easily seen that $a v$ is of maximal rank in $\operatorname{Soc}(A)$. By the diagonalisation theorem ([3, Theorem 2.8]) it follows that $a v$ is group invertible. If $b$ is the group inverse for $a v$ then, in view of $1.6, b$ commutes with both $a$ and $v$. It follows by the invertibility of $v$ that $v b$ is the group inverse of $a$.

By definition, the set of maximal finite rank elements is contained in the set $R=\{a \in \operatorname{Soc}(A): a$ assumes its rank on $\operatorname{comm}(a)\}$ and hence $R$ is also dense in $\operatorname{Soc}(A)$. However, it is easy to see that the containment is strict in general. On the other hand, consideration of matrix algebras shows that Proposition 2.1 does not characterize elements of $\operatorname{Soc}(A)$ belonging to $\mathcal{G}(A)$. Our next result is an extension of [5, Theorem 11].

Corollary 2.2. The following conditions on $\operatorname{Soc}(A)$ are equivalent:

(i) $\operatorname{Soc}(A) \subseteq \mathcal{G}(A)$,

(ii) every element of $\operatorname{Soc}(A)$ is central in $A$,

(iii) every $a \in \operatorname{Soc}(A)$ assumes its rank on $\operatorname{comm}(a)$,

(iv) 0 is the only nilpotent element in $\operatorname{Soc}(A)$,

(v) the distance between any two distinct idempotents in $\operatorname{Soc}(A)$ is at least one,

(vi) the set of rank 1 idempotents is orthogonal.

Proof. The equivalence of (i) and (ii) was shown in [5]. Obviously (ii) implies (iii), and (iii) implies (i) by Proposition 2.1. If 0 is the only nilpotent element in $\operatorname{Soc}(A)$ then, by the core-nilpotent decomposition, $\operatorname{Soc}(A) \subseteq$ $\mathcal{G}(A)$. Conversely, if $a \in \operatorname{Soc}(A)$ is group invertible and $a^{k}=0$ for some $k \in \mathbb{N}$ then, since $a$ and $a^{D}$ commute, we have $\sigma\left(a a^{D}\right)=\{0\}$, which implies $a a^{D}=0$ because $a a^{D}$ is an idempotent. Thus $a a^{D} a=a=0$. 
Suppose (ii) holds. If $p$ and $q$ are distinct idempotents in $\operatorname{Soc}(A)$ then it follows that $(p-q)^{3}=p-q$ and hence that $\|p-q\| \geq 1$. Thus (ii) implies (v). On the other hand, if $(\mathrm{v})$ holds then, since $\operatorname{Soc}(A)$ is a two-sided ideal, it follows that, for an idempotent $p \in \operatorname{Soc}(A)$ and $x \in A$ arbitrary, $e^{\lambda x} p e^{-\lambda x} \in$ $\operatorname{Soc}(A)$ for each $\lambda \in \mathbb{C}$. By continuity of the map $\lambda \mapsto e^{\lambda x} p e^{-\lambda x}-p$ there exists an open ball $B_{0}=B(0, \varepsilon), \varepsilon>0$, in $\mathbb{C}$ such that $\left\|e^{\lambda x} p e^{-\lambda x}-p\right\|<1$ for all $\lambda \in B_{0}$. So, using the assumption in (v), $e^{\lambda x} p e^{-\lambda x}-p=0$ for all $\lambda \in B_{0}$. Since the function $\lambda \mapsto e^{\lambda x} p e^{-\lambda x}-p$ is also analytic on $\mathbb{C}$ it follows by the scarcity theorem for the rank $\left(\left[3\right.\right.$, Theorem 2.3]) that $\operatorname{rank}\left(e^{\lambda x} p e^{-\lambda x}-p\right)=0$ for all $\lambda \in \mathbb{C}$. So, for $\lambda=1$, we have $e^{x} p=p e^{x}$, that is, $p$ commutes with every element of $\exp (A)$. Since $\exp (A)+\exp (A)=A$ it follows that $p$ is central in $A$. The implication (v) $\Rightarrow($ ii) now follows from the density of the maximal finite rank elements in $\operatorname{Soc}(A)$.

If (ii) holds and $p, q$ are nonzero rank 1 idempotents then $p q \neq 0$ implies $p q=p=q$ using the minimality of $p$ and $q$ together with Theorem 1.2. Thus (ii) implies (vi). Finally, if (vi) holds then let $p$ be a rank 1 idempotent and let $a \in A$ be arbitrary. If we choose $\alpha, \beta \in \mathbb{C}$ such that $\alpha, \beta$ belong to the resolvent of $a$ with $\alpha \neq \beta$ then $(\alpha-a) p(\alpha-a)^{-1}$ and $(\beta-a) p(\beta-a)^{-1}$ are also rank 1 idempotents. If neither of them equals $p$ then, by assumption, their respective products with $p$ are zero. But this yields $\alpha p=p a p=\beta p$, which is a contradiction. Hence $p=(\alpha-a) p(\alpha-a)^{-1}$ for some $\alpha$ in the resolvent of $a$, from which one obtains $p a=a p$. Again, by the density of the maximal finite rank elements, (ii) follows.

As opposed to Corollary 2.2 we now identify those elements of $\operatorname{Soc}(A)$ belonging to $\mathcal{G}(A)$ which, together with Lemmas 2.5 and 2.6, lead to a connection between the Drazin index and the rank, thus generalizing the case $A=M_{n}(\mathbb{C})([9$, p. 126]) to the socle of an arbitrary semisimple Banach algebra.

TheOREm 2.3. If $a \in \operatorname{Soc}(A)$ with Drazin inverse $a^{D}$ then $a \in \mathcal{G}(A)$ if and only if $\operatorname{rank}(a)=\operatorname{rank}\left(a^{D}\right)$.

Proof. $\Rightarrow$ If $a$ is group invertible with group inverse $a^{D}$ then

$$
\operatorname{rank}(a)=\operatorname{rank}\left(a^{2} a^{D}\right) \leq \operatorname{rank}\left(a^{D}\right)=\operatorname{rank}\left(a\left(a^{D}\right)^{2}\right) \leq \operatorname{rank}(a) .
$$

Thus $\operatorname{rank}(a)=\operatorname{rank}\left(a^{D}\right)$.

$\Leftarrow \operatorname{Suppose} \operatorname{rank}(a)=\operatorname{rank}\left(a^{D}\right)$. First note that since $a^{D}$ is group invertible with group inverse $a a^{D} a$, they are both invertible in the semisimple algebra $a a^{D} A a a^{D}$ (which has $a a^{D}$ as identity). Hence

$$
\operatorname{rank}(a)=\operatorname{rank}\left(a^{D}\right)=\operatorname{rank}\left(a a^{D} a\right)=\operatorname{rank}\left(a a^{D}\right) .
$$

Choose $x \in E\left(a a^{D} a\right)$ and $y \in E\left(\left(1-a a^{D}\right) a\right)$ such that

$$
\sigma\left(a a^{D} a x\right) \cap \sigma\left(\left(1-a a^{D}\right) a y\right)=\{0\} .
$$


Since $a$ and $a^{D}$ commute we have

$$
\begin{aligned}
\operatorname{rank}(a) & \geq \operatorname{rank}\left(a a^{D} a x a a^{D}+\left(1-a a^{D}\right) a y\left(1-a a^{D}\right)\right) \\
& \geq \# \sigma^{\prime}\left(a a^{D} a x a a^{D}+\left(1-a a^{D}\right) a y\left(1-a a^{D}\right)\right) \\
& =\#\left[\sigma^{\prime}\left(a a^{D} a x a a^{D}\right) \cup \sigma^{\prime}\left(\left(1-a a^{D}\right) a y\left(1-a a^{D}\right)\right)\right] \\
& =\# \sigma^{\prime}\left(a a^{D} a x a a^{D}\right)+\# \sigma^{\prime}\left(\left(1-a a^{D}\right) a y\left(1-a a^{D}\right)\right) \\
& =\operatorname{rank}\left(a a^{D} a\right)+\operatorname{rank}\left(\left(1-a a^{D}\right) a\right) \\
& =\operatorname{rank}(a)+\operatorname{rank}\left(\left(1-a a^{D}\right) a\right) .
\end{aligned}
$$

Thus, since the rank is nonnegative, we have $\operatorname{rank}\left(\left(1-a a^{D}\right) a\right)=0$. It follows that $\left(1-a a^{D}\right) a=0$ and hence $a$ is group invertible.

TheOREM 2.4. An element $a \in \operatorname{Soc}(A)$ belongs to $\mathcal{G}(A)$ if and only if $\operatorname{rank}(a)=\operatorname{rank}\left(a^{k}\right)$ for each $k \in \mathbb{N}$.

Proof. $\Rightarrow$ If $a$ is group invertible with group inverse $b$ then, obviously, for each $k \in \mathbb{N}, a^{k}$ is group invertible with group inverse $b^{k}$. But all these elements are invertible in the semisimple algebra $a b A a b$ and hence all of them have rank equal to $\operatorname{rank}(a b)$.

$\Leftarrow \operatorname{Suppose} \operatorname{rank}(a)=\operatorname{rank}\left(a^{k}\right)$ for each $k \in \mathbb{N}$ and suppose $a$ has Drazin index $k_{1} \in \mathbb{N}$. If $b$ is the Drazin inverse of $a$ then $a^{k_{1}}$ is group invertible with group inverse $b^{k_{1}}$. But clearly, since $b$ is group invertible, we have $\operatorname{rank}(b)=$ $\operatorname{rank}\left(b^{k_{1}}\right)$ by the first part of the proof. So, from $\operatorname{rank}\left(a^{k_{1}}\right)=\operatorname{rank}\left(b^{k_{1}}\right)$, it follows that $\operatorname{rank}(a)=\operatorname{rank}(b)$. By Theorem 2.3, $a$ is group invertible.

Lemma 2.5. The Drazin index of $a \in \operatorname{Soc}(A)$ is the least nonnegative integer $k$ such that $a^{k}$ is group invertible.

Proof. If the Drazin index of $a$ is $k$ then it is clear that $a^{k}$ is group invertible. Now suppose $a^{j}$ is also group invertible for some $j \in \mathbb{N}$ with $j<k$. Denote by $b$ the Drazin inverse of $a$ and by $c$ the group inverse of $a^{j}$. By the core-nilpotent decomposition we have $a^{j}=(a b a)^{j}+a^{j}(1-a b)$ and also $a^{j}=a^{j} c a^{j}+0$. It follows from the uniqueness of the decomposition that $a^{j}(1-a b)=0$. But this contradicts the definition of the Drazin index, that is, that $k$ is the least nonnegative integer such that $a^{k} b a=a^{k}$.

LEMma 2.6. For $a \in \operatorname{Soc}(A)$ the rank of a is additive on the rank structure decomposition of $a$.

Proof. Let $a \in \operatorname{Soc}(A)$ with rank structure decomposition $a=\sum_{i=1}^{m} a_{i}$. Then clearly $\operatorname{rank}(a)=\operatorname{rank}\left(\sum_{i=1}^{m} \lambda_{i} a_{i}\right)$ for each choice $\lambda_{1}, \ldots, \lambda_{m}$ of nonzero scalars. Choose $x \in E(a) \cap E\left(a_{1}\right) \cap \cdots \cap E\left(a_{m}\right)$ such that $\operatorname{rank}(a)=$ $\# \sigma^{\prime}(a x)=\# \sigma^{\prime}\left(\sum_{i=1}^{m} a_{i} x\right)$. Obviously we may choose $\lambda_{1}, \ldots, \lambda_{m}$ such that 
$\sigma\left(\lambda_{i} a_{i} x\right) \cap \sigma\left(\lambda_{j} a_{j} x\right)=\{0\}$ for all $i \neq j$. It follows that

$$
\begin{aligned}
\operatorname{rank}(a) & \geq \# \sigma^{\prime}\left(\sum_{i=1}^{m} \lambda_{i} a_{i} x\right)=\#\left[\bigcup_{i=1}^{m} \sigma^{\prime}\left(\lambda_{i} a_{i} x\right)\right]=\sum_{i=1}^{m} \# \sigma^{\prime}\left(\lambda_{i} a_{i} x\right) \\
& =\sum_{i=1}^{m} \# \sigma^{\prime}\left(a_{i} x\right)=\sum_{i=1}^{m} \operatorname{rank}\left(a_{i}\right) \geq \operatorname{rank}(a)
\end{aligned}
$$

where the final inequality follows from the subadditivity of the rank ([3, Theorem 2.14]). Thus $\operatorname{rank}(a)=\sum_{i=1}^{m} \operatorname{rank}\left(a_{i}\right)$.

TheOREM 2.7. An element $a \in \operatorname{Soc}(A)$ has Drazin index $k$ if and only if $k$ is the least integer satisfying

$$
\operatorname{rank}\left(a^{k}\right)=\operatorname{rank}\left(a^{k+1}\right) .
$$

In particular, a is group invertible if and only if $\operatorname{rank}(a)=\operatorname{rank}\left(a^{2}\right)$.

Proof. $\Leftarrow$ Let $a \in \operatorname{Soc}(A)$ and suppose $k$ is the least nonnegative integer such that $\operatorname{rank}\left(a^{k}\right)=\operatorname{rank}\left(a^{k+1}\right)$. If $a=\sum_{i=1}^{m} a_{i}$ is the rank structure decomposition of $a$ then $a^{k}=\sum_{i=1}^{m} a_{i}^{k}$ and $a^{k+1}=\sum_{i=1}^{m} a_{i}^{k+1}$ are the rank structure decompositions of $a^{k}$ and $a^{k+1}$ respectively (see [4, Proposition 2.4]). By Lemma 2.6 it follows that

$$
\operatorname{rank}\left(a^{k}\right)=\sum_{i=1}^{m} \operatorname{rank}\left(a_{i}^{k}\right)=\operatorname{rank}\left(a^{k+1}\right)=\sum_{i=1}^{m} \operatorname{rank}\left(a_{i}^{k+1}\right) .
$$

Now since $\operatorname{rank}\left(a_{i}^{k}\right) \geq \operatorname{rank}\left(a_{i}^{k+1}\right)$ for each $i$ we actually have $\operatorname{rank}\left(a_{i}^{k}\right)=$ $\operatorname{rank}\left(a_{i}^{k+1}\right)$ for each $i$. It follows from Theorem 1.1 that

$$
a_{i}^{k} A a_{i}^{k} \simeq M_{r_{i}, n_{i}} \quad \text { and } \quad a_{i}^{k+1} A a_{i}^{k+1} \simeq M_{r_{i}, n_{i}^{\prime}}
$$

where $n_{i}^{\prime} \leq n_{i}$. Note that $r_{i}$ is the same for $a_{i}^{k}$ and $a_{i}^{k+1} \operatorname{since} \operatorname{rank}\left(a_{i}^{k}\right)=$ $\operatorname{rank}\left(a_{i}^{k+1}\right)$. Hence the dimension of $a_{i}^{k+1} A a_{i}^{k+1}$ does not diminish, that is,

$$
\operatorname{dim}\left(a_{i}^{k+1} A a_{i}^{k+1}\right)=\operatorname{dim}\left(a_{i}^{k} A a_{i}^{k}\right)
$$

Since $a_{i}^{k+1} A a_{i}^{k+1}$ is a vector subspace of $a_{i}^{k} A a_{i}^{k}$ we have $a_{i}^{k+1} A a_{i}^{k+1}=$ $a_{i}^{k} A a_{i}^{k}$. Inductively it follows that $a_{i}^{k+r} A a_{i}^{k+r}=a_{i}^{k} A a_{i}^{k}$ for each $r \in \mathbb{N}$. Thus by Theorem 1.1, $\operatorname{rank}\left(a_{i}^{k}\right)=\operatorname{rank}\left(a_{i}^{k+r}\right)$ for each $r \in \mathbb{N}$. This being true for all $i$, we have $\operatorname{rank}\left(a^{k}\right)=\operatorname{rank}\left(a^{k+r}\right)$ for each $r \in \mathbb{N}$. In particular $\operatorname{rank}\left(a^{k}\right)=\operatorname{rank}\left(\left(a^{k}\right)^{n}\right)$ for all $n \in \mathbb{N}$. So, using Theorem 2.4, we infer that $a^{k}$ is group invertible. Also, if $j<k$ then, by assumption, we have

$$
\operatorname{rank}\left(a^{j}\right)>\operatorname{rank}\left(a^{j+1}\right) \geq \cdots \geq \operatorname{rank}\left(\left(a^{j}\right)^{2}\right)
$$

and hence $a^{j}$ cannot be group invertible. So if $k$ is the least integer such that $\operatorname{rank}\left(a^{k}\right)=\operatorname{rank}\left(a^{k+1}\right)$ then $k$ is the least integer such that $a^{k}$ is group invertible. By Lemma 2.5, $k$ is the Drazin index of $a$. 
$\Rightarrow$ If $a$ has Drazin index $k$ then by Lemma 2.5, $k$ is the least integer such that $a^{k}$ is group invertible. Hence, as in the first part of the proof, we cannot have $\operatorname{rank}\left(a^{k}\right)>\operatorname{rank}\left(a^{k+1}\right) . \operatorname{Thus} \operatorname{rank}\left(a^{k}\right)=\operatorname{rank}\left(a^{k+1}\right)$.

Corollary 2.8. If $a b$ and ba belong to $\operatorname{Soc}(A)$ and $a b \in \mathcal{G}(A)$ then $b a \in \mathcal{G}(A)$ if and only if $\operatorname{rank}(a b)=\operatorname{rank}(b a)$.

Proof. $\Leftarrow$ If $a b$ is group invertible then by Theorem $2.4, \operatorname{rank}\left((a b)^{k}\right)=$ $\operatorname{rank}(a b)$ for each $k \in \mathbb{N}$. So if $\operatorname{rank}(a b)=\operatorname{rank}(b a)$ then, for $k>1$,

$$
\begin{aligned}
\operatorname{rank}(b a) & =\operatorname{rank}(a b)=\operatorname{rank}\left((a b)^{k}\right)=\operatorname{rank}(\underbrace{a b a b \ldots a b}_{k \text { times }}) \\
& \leq \operatorname{rank}(\underbrace{b a b a \ldots b a}_{k-1 \text { times }})=\operatorname{rank}\left((b a)^{k-1}\right) \leq \operatorname{rank}(b a) .
\end{aligned}
$$

Since $k>1$ was arbitrary the result follows by Theorem 2.4 .

$\Rightarrow$ If $a b$ and $b a$ are both group invertible then, by Theorem 2.7,

$$
\operatorname{rank}(a b)=\operatorname{rank}(a b a b) \leq \operatorname{rank}(b a)=\operatorname{rank}(b a b a) \leq \operatorname{rank}(a b) .
$$

Hence $\operatorname{rank}(a b)=\operatorname{rank}(b a)$.

In [5] it was shown that $1-a b$ is Drazin invertible if and only if $1-b a$ is Drazin invertible, with preservation of the index. This is not true for $a b$ and $b a$ : Consider a Banach algebra $A$ with $a, b \in A$ satisfying $a b=1$ but $b a \neq 1$. Then $a b$ is Drazin invertible with Drazin index 0 but, since $b a$ is not invertible, $b a$ cannot have Drazin index equal to 0. However, using the fact that $a b=1$, it is easy to see that $b a$ is an idempotent and thus group invertible. So $b a$ is also Drazin invertible but with Drazin index 1. Generally speaking this is the worst that could happen: it is not hard to prove, using the definition of the Drazin inverse, that if $a b$ is Drazin invertible then $b a$ is always Drazin invertible, with the respective indices differing by at most one.

Much of the existing theory of Drazin invertibility for matrix algebras (see [6, Chapter 7]) is derived through the use of matrix block forms. Now many more of these results may be generalized to the Banach algebra case (not only to socle elements) using the functional calculus representation (1.6). We only give one more example (thereby generalizing [6, Corollary 7.6.2(ii)]), suggesting how one may proceed.

Theorem 2.9. Let $a \in A$ be Drazin invertible. Then a has Drazin index $k$ if and only if $k$ is the least integer such that

exists.

$$
\lim _{\lambda \rightarrow 0}(a-\lambda)^{-1} a^{k}
$$

Proof. $\Rightarrow$ Suppose $a$ is Drazin invertible with Drazin index $k$. Let $U_{0}$ and $U_{1}$ be separated open sets in $\mathbb{C}$ such that $0 \in U_{0}$ and $\sigma^{\prime}(a) \subseteq U_{1}$. Let $\left(\lambda_{n}\right)$ 
be a sequence in $\mathbb{C}$ such that $\left(\lambda_{n}\right) \subseteq U_{0}$ and $\lambda_{n} \rightarrow 0$. For each $n$ let $V_{n} \subseteq U_{0}$ be an open ball with center 0 such that $\lambda_{n} \notin V_{n}$. Let $\Gamma$ be a smooth contour in $U_{1}$ surrounding $\sigma^{\prime}(a)$ and let $\Gamma_{0}$ be a small circle in $U_{0}$ surrounding 0 . Finally, for each $n$, let $\Gamma_{n}$ be a small circle in $V_{n}$ surrounding 0 . Now, for each $n, a-\lambda_{n}$ is invertible and

$$
\left(a-\lambda_{n}\right)^{-1}=\frac{1}{2 \pi i} \int_{\Gamma \cup \Gamma_{n}}\left(\alpha-\lambda_{n}\right)^{-1}(\alpha-a)^{-1} d \alpha .
$$

Also, using the fact that $a^{k+1} a^{D}=a^{k}$, we find that

$$
a^{k}=\frac{1}{2 \pi i} \int_{\Gamma \cup \Gamma_{0}} f(\alpha)(\alpha-a)^{-1} d \alpha=\frac{1}{2 \pi i} \int_{\Gamma \cup \Gamma_{n}} f_{n}(\alpha)(\alpha-a)^{-1} d \alpha
$$

for each $n$, where $f$ is defined by

$$
f(\alpha)= \begin{cases}\alpha^{k}, & \alpha \in U_{1}, \\ 0, & \alpha \in U_{0},\end{cases}
$$

and $f_{n}$ is the restriction of $f$ to $V_{n} \cup U_{1}$. It follows by the holomorphic functional calculus that, for each $n$,

$$
\left(a-\lambda_{n}\right)^{-1} a^{k}=\frac{1}{2 \pi i} \int_{\Gamma \cup \Gamma_{n}} \frac{f_{n}(\alpha)}{\alpha-\lambda_{n}}(\alpha-a)^{-1} d \alpha=\frac{1}{2 \pi i} \int_{\Gamma \cup \Gamma_{0}} g_{n}(\alpha)(\alpha-a)^{-1} d \alpha
$$

where $g_{n}$ is defined by

$$
g_{n}(\alpha)= \begin{cases}\frac{\alpha^{k}}{\alpha-\lambda_{n}}, & \alpha \in U_{1} \\ 0, & \alpha \in U_{0}\end{cases}
$$

If we define $g$ by

$$
g(\alpha)= \begin{cases}\alpha^{k-1}, & \alpha \in U_{1}, \\ 0, & \alpha \in U_{0},\end{cases}
$$

then $\left(g_{n}\right)$ is a sequence of holomorphic functions on $U_{0} \cup U_{1}$, converging uniformly to $g$ on compact subsets of $U_{0} \cup U_{1}$. We hence have

$$
\begin{aligned}
\lim _{n}\left(a-\lambda_{n}\right)^{-1} a^{k} & =\lim _{n} \frac{1}{2 \pi i} \int_{\Gamma \cup \Gamma_{0}} g_{n}(\alpha)(\alpha-a)^{-1} d \alpha \\
& =\frac{1}{2 \pi i} \int_{\Gamma \cup \Gamma_{0}} g(\alpha)(\alpha-a)^{-1} d \alpha=a^{k} a^{D} .
\end{aligned}
$$

Thus, if the Drazin index of $a$ is $k$, then $k$ is an integer such that the limit $\lim _{\lambda \rightarrow 0}(a-\lambda)^{-1} a^{k}$ exists. Suppose now that $\lim _{\lambda \rightarrow 0}(a-\lambda)^{-1} a^{l}=b$ exists for some $l<k$. Then it follows easily that $a^{l}=a b=b a$ and also that

$$
\lim _{\lambda \rightarrow 0}(a-\lambda)^{-1} a^{l} a^{k-l}=b a^{k-l}=a^{k} a^{D} .
$$


Hence $a^{l} a^{k-l-1}=b a a^{k-l-1}=b a^{k-l}=a^{k} a^{D}$, from which we obtain $a^{k-1}=$ $a^{k} a^{D}$. But since $a^{k} a^{D}$ is group invertible we have a contradiction, because $k$ is the least integer such that $a^{k}$ is group invertible.

$\Leftarrow$ Suppose $k$ is the least integer such that $\lim _{\lambda \rightarrow 0}(a-\lambda)^{-1} a^{k}$ exists, but that $a$ has Drazin index $l<k$. By the first part of the proof it follows that $\lim _{\lambda \rightarrow 0}(a-\lambda)^{-1} a^{l}=a^{l} a^{D}$, which contradicts our assumption. Thus, under the above hypothesis, $a$ must have Drazin index $k$.

3. Rank and continuity. Continuity properties of the Drazin inverse were studied by several authors ([6], [8], [10], [11]), and in particular Koliha and Rakočević obtained an operator-theoretic generalization ([8, Theorem 5.1]) of a well known criterion ([6, Theorem 10.7.1]) for continuity of the Drazin inverse for matrices. Of course, their result implies Campbell and Meyer's theorem. We show here (Corollary 3.4) that with Aupetit and Mouton's spectral rank characterization, Campbell and Meyer's result can be extended to the socle of an arbitrary semisimple Banach algebra. We shall need the following lemma and then first prove the special case for group invertibility:

Lemma 3.1. Let $A$ be a semisimple Banach algebra. If $a_{n}, a \in \operatorname{Soc}(A)$ are maximal rank with $a_{n} \rightarrow a$ and there exists $n_{0} \in \mathbb{N}$ such that $\operatorname{rank}\left(a_{n}\right)=$ $\operatorname{rank}(a)$ for $n \geq n_{0}$ then $a_{n}^{D} \rightarrow a^{D}$.

Proof. For $n \geq n_{0}$ we have $\operatorname{rank}\left(a_{n}\right)=\# \sigma^{\prime}\left(a_{n}\right)=\# \sigma^{\prime}(a)=\operatorname{rank}(a)$. By continuity of the spectrum on $\operatorname{Soc}(A)$ we have $\inf _{n} d\left(0, \sigma^{\prime}\left(a_{n}\right)\right)>0$. It follows from [8, Theorem 2.4] that $a_{n}^{D} \rightarrow a^{D}$.

THEOREM 3.2. Let $a_{n}$ and $a$ be group invertible elements belonging to $\operatorname{Soc}(A)$ with $a_{n} \rightarrow a$. Then $a_{n}^{D} \rightarrow a^{D}$ if and only if there exists $n_{0} \in \mathbb{N}$ such that $\operatorname{rank}\left(a_{n}\right)=\operatorname{rank}(a)$ for all $n \geq n_{0}$.

Proof. $\Rightarrow$ If $a_{n}^{D} \rightarrow a^{D}$ then $a_{n} a_{n}^{D} \rightarrow a a^{D}$ and hence by [11, Lemma 12] for some $n_{0} \in \mathbb{N}$ the elements $a_{n} a_{n}^{D}$ and $a a^{D}$ are similar for $n \geq n_{0}$, that is, there are $x_{n} \in A^{-1}$ such that $x_{n} a_{n} a_{n}^{D} x_{n}^{-1}=a a^{D}$. By the rank definition $\operatorname{rank}\left(a_{n} a_{n}^{D}\right)=\operatorname{rank}\left(a a^{D}\right)$ for $n \geq n_{0}$. A simple argument, using $a_{n}=a_{n} a_{n}^{D} a_{n}$ and $a=a a^{D} a$, implies $\operatorname{rank}\left(a_{n}\right)=\operatorname{rank}(a)$ for $n \geq n_{0}$.

$\Leftarrow$ Set the idempotents $a_{n} a_{n}^{D}=q_{n}$ and $a a^{D}=q$ and consider the finite-dimensional, semisimple and unital Banach algebras $B_{n}=q_{n} A q_{n}$ and $B=q A q$. Using Baire's theorem we can find $x \in A$ such that $\operatorname{rank}\left(a_{n}\right)=$ $\# \sigma^{\prime}\left(a_{n} x\right)=\# \sigma^{\prime}(a x)=\operatorname{rank}(a)$. Now, for $n \geq n_{0}$ we have

$$
\operatorname{rank}\left(a_{n}\right)=\# \sigma^{\prime}\left(a_{n}\left(q_{n} x q_{n}\right)\right)=\# \sigma^{\prime}(a(q x q))=\operatorname{rank}(a) .
$$

By the above equation and the fact that $a_{n}$ and $a$ are invertible in the algebras $B_{n}$ and $B$ respectively, the elements $q_{n} x q_{n}$ and $q x q$ are also invertible in $B_{n}$ and $B$ (for all $n \geq n_{0}$ ). Moreover, the inverses of $q_{n} x q_{n}$ and $q x q$ in 
$B_{n}$ and $B$ are just the Drazin (group) inverses $\left(q_{n} x q_{n}\right)^{D}$ and $(q x q)^{D}$. We now show that $q_{n} x q_{n} \rightarrow q x q$ and that $\left(q_{n} x q_{n}\right)^{D} \rightarrow(q x q)^{D}$ :

Since $a_{n} \rightarrow a$ we have $a_{n} x a_{n}=a_{n}\left(q_{n} x q_{n}\right) a_{n} \rightarrow a(q x q) a=a x a$. Since $a_{n}\left(q_{n} x q_{n}\right) a_{n}$ and $a(q x q) a$ are maximal rank $\left(n \geq n_{0}\right)$ it follows from Lemma 3.1 that $\left[a_{n}\left(q_{n} x q_{n}\right) a_{n}\right]^{D} \rightarrow[a(q x q) a]^{D}$. By the invertibility of $a_{n}$ and $q_{n} x q_{n}$ in $B_{n}$ and of $a$ and $q x q$ in $B$ we can apply the inverse formula with $D$, that is, $a_{n}^{D}\left(q_{n} x q_{n}\right)^{D} a_{n}^{D} \rightarrow a^{D}(q x q)^{D} a^{D}$. Now, multiplying by $a_{n}$ and $a$ on both sides yields $a_{n} a_{n}^{D}\left(q_{n} x q_{n}\right)^{D} a_{n}^{D} a_{n} \rightarrow a a^{D}(q x q)^{D} a^{D} a$, which implies $\left(q_{n} x q_{n}\right)^{D} \rightarrow(q x q)^{D}$ because $a_{n} a_{n}^{D}$ and $a a^{D}$ are the identities in $B_{n}$ and $B$ respectively. From Lemma 3.1 together with $\left[\left(q_{n} x q_{n}\right)^{D}\right]^{D}=q_{n} x q_{n}$ and $\left[(q x q)^{D}\right]^{D}=q x q$ it follows that $q_{n} x q_{n} \rightarrow q x q$. To complete the proof notice that $\left(q_{n} x q_{n}\right)\left(q_{n} x q_{n}\right)^{D}=a_{n} a_{n}^{D}$ and $q x q(q x q)^{D}=a a^{D}$, so that $a_{n} a_{n}^{D} \rightarrow a a^{D}$. The result now follows from [10, Theorem 4.1].

COROllary 3.3. Let $a_{n}, a \in \operatorname{Soc}(A)$ be such that $a_{n} \rightarrow a$ and suppose the Drazin indices of the $a_{n}$ 's are bounded. Denote by $c_{a_{n}}$ and $c_{a}$ the core elements of $a_{n}$ and a respectively. Then $a_{n}^{D} \rightarrow a^{D}$ if and only if there exists $n_{0} \in \mathbb{N}$ such that $\operatorname{rank}\left(c_{a_{n}}\right)=\operatorname{rank}\left(c_{a}\right)$ for all $n \geq n_{0}$.

Proof. $\Rightarrow$ Since $\operatorname{rank}\left(c_{a}=a a^{D} a\right)=\operatorname{rank}\left(a a^{D}\right)$ for socle elements, the result follows by consideration of the first part of the proof of Theorem 3.2.

$\Leftarrow$ Let $k_{n}, k$ denote the Drazin indices of $a_{n}, a$. Since $k_{n}, k$ are bounded there exists $m \in \mathbb{N}$ such that $a_{n}^{m}, a^{m}$ are group invertible. So, as $\operatorname{rank}\left(c_{a_{n}}\right)=$ $\operatorname{rank}\left(c_{a}\right)$ for all $n \geq n_{0}$, we have $\operatorname{rank}\left(a_{n}^{m}\right)=\operatorname{rank}\left(a^{m}\right)$ for $n \geq n_{0}$. From Theorem 3.2 we obtain $\left(a_{n}^{m}\right)^{D} \rightarrow\left(a^{m}\right)^{D}$ and since $a_{n}^{m} \rightarrow a^{m}$ it follows that $a_{n} a_{n}^{D} \rightarrow a a^{D}$. Thus [10, Theorem 4.1] implies $a_{n}^{D} \rightarrow a^{D}$.

\section{References}

[1] B. Aupetit, Spectrum-preserving linear mappings between Banach algebras or Jordan-Banach algebras, J. London Math. Soc. (2) 62 (2000), 917-924.

[2] - - A Primer on Spectral Theory, Springer, 1991.

[3] B. Aupetit and H. du T. Mouton, Trace and determinant in Banach algebras, Studia Math. 121 (1996), 115-136.

[4] M. Brešar and P. Šemrl, Finite rank elements in semisimple Banach algebras, ibid. 128 (1998), 287-298.

[5] R. Brits, L. Lindeboom and H. Raubenheimer, On ideals of generalized invertible elements in Banach algebras, Math. Proc. Roy. Irish Acad. Sect. A 105 (2005), 1-10.

[6] S. L. Campbell and C. D. Meyer, Generalized Inverses of Linear Transformations, Dover, New York, 1991.

[7] J. J. Koliha, A generalized Drazin inverse, Glasgow Math. J. 38 (1996), 367-381.

[8] J. J. Koliha and V. Rakočević, Continuity of the Drazin inverse II, Studia Math. 131 (1998), 167-177.

[9] M. Z. Nashed, Generalized Inverses and Applications, Academic Press, New York, 1976 . 
[10] V. Rakočević, Continuity of the Drazin inverse, J. Operator Theory 41 (1999), 55-68.

[11] S. Roch and B. Silberman, Continuity of generalized inverses in Banach algebras, Studia Math. 136 (1999), 197-227.

Department of Mathematics

University of Johannesburg

P.O. Box 524

Auckland Park 2006, South Africa

E-mail: rbri@rau.ac.za

hra@rau.ac.za

Department of Mathematics, Applied Mathematics and Astronomy

University of South Africa

P.O. Box 392

UNISA 0003, Pretoria, South Africa

E-mail: lindel@unisa.ac.za

Received August 30, 2004

Revised version November 7, 2006 